Frédéric Grillot, Dejan Arsenijevic, Heming Huang, Dieter Bimberg

\title{
Ultrafast and nonlinear dynamics of InAs/ GaAs semiconductor quantum dot lasers
}

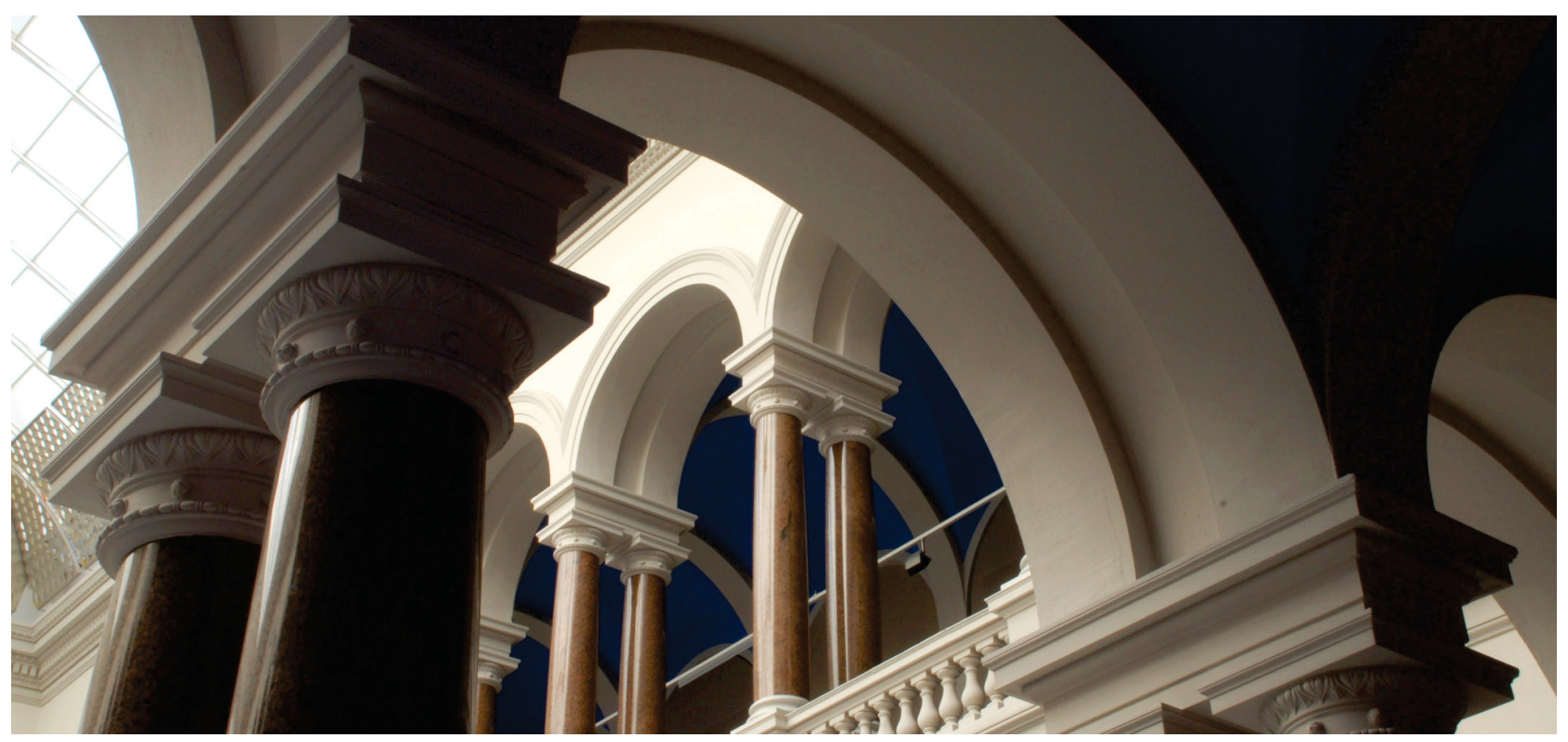

Grillot, F., Arsenijevic, D., Bimberg, D., \& Huang, H. (2018). Ultrafast and nonlinear dynamics of InAs/ GaAs semiconductor quantum dot lasers. Proc. SPIE 10543, Quantum Dots and Nanostructures: Growth, Characterization, and Modeling XV, 105430M (21 February 2018). https://doi.org/10.1117/12.2299678. 


\title{
Ultrafast and nonlinear dynamics of InAs/GaAs semiconductor quantum dot lasers
}

\author{
F. Grillott ${ }^{\mathrm{a}, \mathrm{b}}$, D. Arsenijevic̀ ${ }^{\mathrm{c}}, \mathrm{H}$. Huang ${ }^{\mathrm{a}}$, and D. Bimberg ${ }^{\mathrm{c}, \mathrm{d}}$ \\ ${ }^{a}$ LTCI, Télécom ParisTech, Université Paris-Saclay, 46 rue Barrault, Paris, France \\ ${ }^{\mathrm{b}}$ Center for High Technology Materials, University of New-Mexico, Albuquerque, USA \\ 'Institut fr Festkrperphysik, Technische Universitt Berlin, Berlin 10623, Germany \\ ${ }^{\mathrm{d} K i n g}$ Abdulaziz University, 22254 Jeddah, Saudi Arabia
}

\begin{abstract}
Quantum dot nanostructures are one of the best practical examples of emerging nanotechnologies hence offering superior properties as compared to their quantum well counterparts. InAs/GaAs quantum dots allow producing energy- and cost-efficient devices with outstanding temperature stability, lowest threshold current, ultrafast gain dynamics, and low amplified spontaneous emission. This paper reports on the recent achievements in ultrafast and nonlinear dynamics properties of InAs/GaAs quantum dot lasers for radar systems, wireless communications and high-speed optical communications. Passive mode-locking is shown to exhibit a great potential for microwave, millimeter-wave and Terahertz signal generation with high repetition frequency tuning and jitter reduction. The optical feedback is also used to stabilize the pulse emission leading an integrated timing jitter as low as 90 fs without consuming additional power. Lastly, multimode optical feedback dynamics of InAs/GaAs QD lasers emitting on different lasing states is also studied. In particular, a chaos free operation is observed for the first time from the ground state lasing operation.
\end{abstract}

Keywords: Quantum dot lasers, passive mode-locking, optical feedback, high-speed communications, radars, wireless communications

\section{INTRODUCTION}

Significant improvements of semiconductor laser have been achieved in the last decades by using zero-dimensional quantum dot $(\mathrm{QD})$ nanostructures as gain media ${ }^{1} .^{2}$ Their inherent properties allow producing energy- and costefficient devices with outstanding temperature stability, low threshold current, ultrafast gain dynamics, and low amplified spontaneous emission. ${ }^{3}$ A low threshold current density and high internal quantum efficiency results in a reduced amount of dissipated heat. Even at high operation temperatures, temperature insensitive threshold currents are observed for p-doped devices. ${ }^{4}$ Most advantages of QD lasers have been demonstrated for GaAsbased ones, operating at $1310 \mathrm{~nm}$, hence mostly targeting short reach communication links such as metro and access networks as well as datacenter optical networks ${ }^{5}{ }^{6}$ In addition to that, self-organized Stranski-Krastanov QD growth leads to an inhomogeneously broadened gain spectra and thus, if all modes are phase-locked, to very narrow pulses and very low transparency current densities per QD layer. Saturable absorbers based on QDs exhibit much faster recovery on the order of $700 \mathrm{fs}$ under large reverse bias, ${ }^{7}$ enabling the generation of subpicosecond pulses at very large frequencies. The timing jitter of mode locked lasers (MLLs) is a result of random fluctuations of the photon density, the gain and the effective refractive index caused by amplified spontaneous emission (ASE). Compared to quantum wells (QWs), QD MLLs exhibit lower levels of ASE, ${ }^{8}$ which reduces the jitter.

In this paper, we report on the ultrafast and nonlinear dynamical properties of InAs/GaAs QD lasers for applications in radar, wireless and high-speed optical data communication systems. First, the dynamics of passively QD MLLs with high repetition frequency tuning and low timing jitter for microwave, millimeter-wave and Terahertz

Further author information: (Send correspondence to F.G.)

F.G.: E-mail: grillot@telecom-paristech.fr 
$(\mathrm{THz})$ signal generation generation are discussed. The direct extraction of the electrical signal from the saturable absorber of a $40 \mathrm{GHz}$ QD MLL is presented. An optical feedback loop is also used to greatly stabilize the pulse emission of passively MLLs. Under proper feedback conditions, a single MLL can exhibit a very low-jitter optical and electrical pulse trains at the same time, thus radically simplifying photonic sources for microwave, millimeter-wave and $\mathrm{THz}$ generation. In a second part, we report on the optical feedback dynamics of two InAs/GaAs QD lasers having identical active regions but emitting exclusively from different energy states. Even if QD lasers are normally engineered to operate on the ground-state (GS) transition because of its lower threshold current density, prior studies have also shown that faster dynamics can be obtained when considering the first excited state (ES). ${ }^{9}$ A proof of concept was achieved with ES QD lasers, for which modulation capabilities up to $25 \mathrm{Gbps}$ (OOK) and 35 Gbps (PAM) have been reported. ${ }^{10}$ In this work, we unveil clear different responses to optical feedback and in particular a chaos-free operation is observed with the GS QD laser which is of paramount importance for the development of future isolator-free optical light sources.

\section{ULTRAFAST DYNAMICS OF QD MODE-LOCKED LASERS}

MLLs emitting electrical and optical pulse trains are meaningful for a variety of applications like $\mathrm{THz}$ photonics $^{11}$ and radio-over-fiber systems. ${ }^{12}$ In high bit-rate optical communication networks, ${ }^{13}$ MLLs are also utilized as optical clocks and, in combination with modulators, as transmitters for optical time-division multiplexing (OTDM) systems. They also find multiple other applications in the communication field, such as for multicarrier generation ${ }^{14}$ and all-optical sampling. ${ }^{15}$ In what follows we first start reviewing the main techniques that are available with semiconductor lasers for microwave, millimeter-wave and $\mathrm{THz}$ signal generation. Then, we investigate the ultra-fast dynamical response of $40 \mathrm{GHz}$ passively MLLs with high repetition frequency tuning and low timing jitter for applications in radar and wireless communication systems.

\section{From microwave to THz signals}

The generation of microwave, millimeter-wave and THz signals with semiconductor lasers can be achieved with multiple optical techniques e.g. optical heterodyne generation, dual-tone lasing, optical injection or mode locking. An extended review is proposed in reference. ${ }^{16}$ Hereinafter, we summarize a few of them.

a. Optical heterodyning consists of using light of two free-running single-mode lasers emitting at different wavelength that is combined and converted to an electrical signal by a detector. The generated photocurrent contains a beat signal at the frequency according to the wavelength distance. This technique enables wide frequency tuneability of the electrical signal, in principle from sub- $\mathrm{MHz}$ to several $\mathrm{THz}$, determined by the wavelength tuneability of the laser sources and the bandwidth of the photodetector. Dual-mode lasers, emitting simultaneously on two different longitudinal resonator modes, provide directly a signal at the frequency equal to the wavelength difference. Compared to two free-running lasers, the tuneability is strongly limited and coarse stepped. In both approaches, the two tones are not necessarily phase-locked meaning that the phase of the generated electrical signal can vary in time.

b. Optically-injected semiconductor lasers are known to exhibit very rich nonlinear dynamical states. In particular, for moderate injection strengths and positive detuning from the Hopf-bifurcation boundary, period-one oscillations of the slave laser cause periodic intensity oscillation of the optical signal. A semiconductor laser operating in period-one oscillation enables generation of a wide-range continuously tunable radio-frequency (RF) signals. However, the phase noise of RF signals generated using the single-tone injection approach is relatively large. By combining optical sideband injection locking and an optical phase-locked loop, a strongly suppressed single-side-band (SSB) phase-noise (PN) power spectral density can be achieved.

c. Mode-locking of semiconductor lasers is also a powerful method. As the locked longitudinal modes of a MLL are equally spaced, a RF signal corresponding to the frequency distance is generated. Additionally, beat signals at higher harmonics are provided as well. The MLL-based approach benefits from low-noise performance, compactness and low power consumption. However, the main drawback is the low frequency tuneability, as the mode spacing is fundamentally determined by the cavity length. By optical filtering of two longitudinal modes of an actively MLL, a millimeter-wave signal can be generated. ${ }^{17}$ Selecting a higher harmonic of a lowfrequency MLL, whereas modes located between the selected ones are suppressed, yields a high-frequency RF 
signal. However, the requirement of a high-power electrical reference source dissolves the low-power consumption advantage of a single laser source.

d. Passive mode-locking does not require electrical sinusoidal signal. The optical pulse train of a passively MLL is converted to an electrical signal at the same frequency by using a high-speed photodetector. A more convenient method is based on the direct extraction of the electrical signal from the saturable absorber of a monolithic passively MLL. The absorber serves as an intra-waveguide photodetector enabling electrical signals to be generated with high conversion efficiencies and simultaneously along with the optical pulse train. When an optical pulse enters the reverse-biased absorber section, free carriers are created. Due to the built-in electric field, the free carriers are swept out of the absorber yielding a pulsed photocurrent. Thus, electrical and optical pulses are generated simultaneously without additional power consumption. Compared to the external detection of the optical pulse train using a high-speed photodiode, the extracted pulses exhibit much larger rise and even longer fall times. The latter is attributed to the limited QW absorber dynamics and the slow carrier escape. To overcome such limitations, passively QD MLLs are greatly preferred because of the sub-picosecond recovery time of the QD absorber. However, the extracted signals still suffer from very high phase-noise levels. Besides, at larger repetition rates, the RF signal generation remains limited by electrical parasitics and require improved $\mathrm{RC}$ time constants of the contact pads as well as shorter absorber lengths. These challenges along with the simultaneous generation of optical pulse trains are discussed in the following.

\section{Passively QD mode-locked lasers}

In this paper, a two-section passively QD MLL is considered. The device represented in Fig. 1 monolithically integrates a saturable absorber whereby both sections are based on the identical epitaxial structure. The AlGaAs/GaAs laser structure is grown by molecular beam epitaxy (MBE). In order to ensure a sufficient gain, the active region consists of $10 \mathrm{InAs}$ QD layers. The emission wavelength of $1310 \mathrm{~nm}$ is achieved by overgrowth of the QDs with an InGaAs layer, referred to as dot-in-a-well structure. P-doping of GaAs spacers between QD layers prefills the hole states and is meant to speed up the recovery of the GS population and to provide temperature insensitivity of the threshold current. ${ }^{18}$ The wafers are then processed into ridge-waveguide edge emitters with stripe width of $4 \mu \mathrm{m}$. The ridge width and particularly the length ratio of the gain to the absorber section (typically ranging from 3:1 to 10:1) are crucial design parameters of monolithic MLLs defining the optical and electrical properties of the pulse train as well as the bias range where complete mode locking occurs. ${ }^{19}$ The two sections are formed by a $20 \mu \mathrm{m}$ gap in the metalization and the upper high-doped contact layer ensuring electrical isolation larger than $10 \mathrm{k} \Omega$. The MLL chips, used here, contain a $900 \mu \mathrm{m}$ long gain section and a 100 $\mu \mathrm{m}$ saturable absorber. The laser facet at the absorber is coated for high reflectivity of $99 \%$, the gain-section facet is as cleaved $(32 \%)$. The fundamental repetition frequency $f$ of the QD MLL, given by $f=c / 2 n L$, where

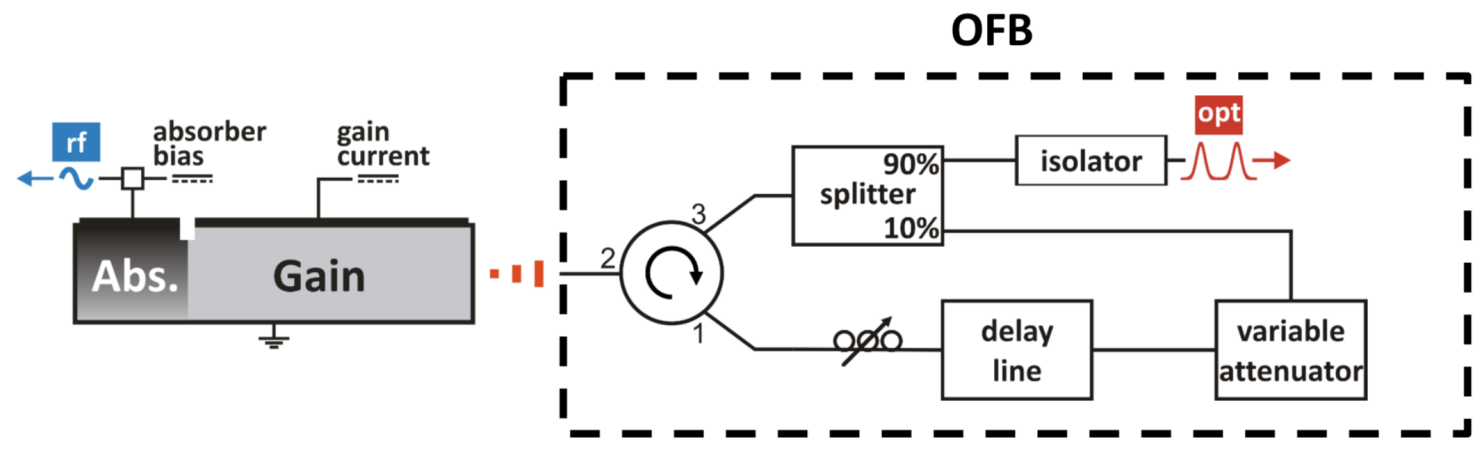

Figure 1. The two-section passively QD MLL with a monolithically integrated saturable absorber. The dashed box represented the optical feedback (OFB) loop described hereinafter.

$c$ is the speed of light in vacuum and $n$ the effective refractive index, equates to the spacing of the longitudinal modes, which in turn is defined by the resonator length $L$. Taking a cavity length of $1 \mathrm{~mm}$ yields a pulse emission at $40 \mathrm{GHz}$. As a consequence of that, the $40-\mathrm{GHz}$ millimeter-wave signal is just located in the IEEE Ka-band $(27-40 \mathrm{GHz})$ as well as in the ITU EHF-band $(30-300 \mathrm{GHz})$ and thus being of great interest for radar systems 
and wireless communications. For the electrical signal, the extraction losses of the experimental setup add up to $4.4 \mathrm{~dB}$. For the present, the absorber impedance is not matched to $50 \Omega$, thus additionally causing coupling losses to the measurement system. Regarding the optical signal, the pulse train is detected using a $50 \mathrm{GHz}$ photodetector.

Fig. 2 depicts a comparison of the RF spectra which reveals a peak frequency of $38.61701 \mathrm{GHz}$ and 38.61706 $\mathrm{GHz}$ for the directly extracted electrical signal and for the one generated from the optical pulse train, respectively. The $50 \mathrm{kHz}$ deviation lies well within the carrier $3-\mathrm{dB}$ linewidth of $187.3 \mathrm{kHz}$. As no photodiode is required,

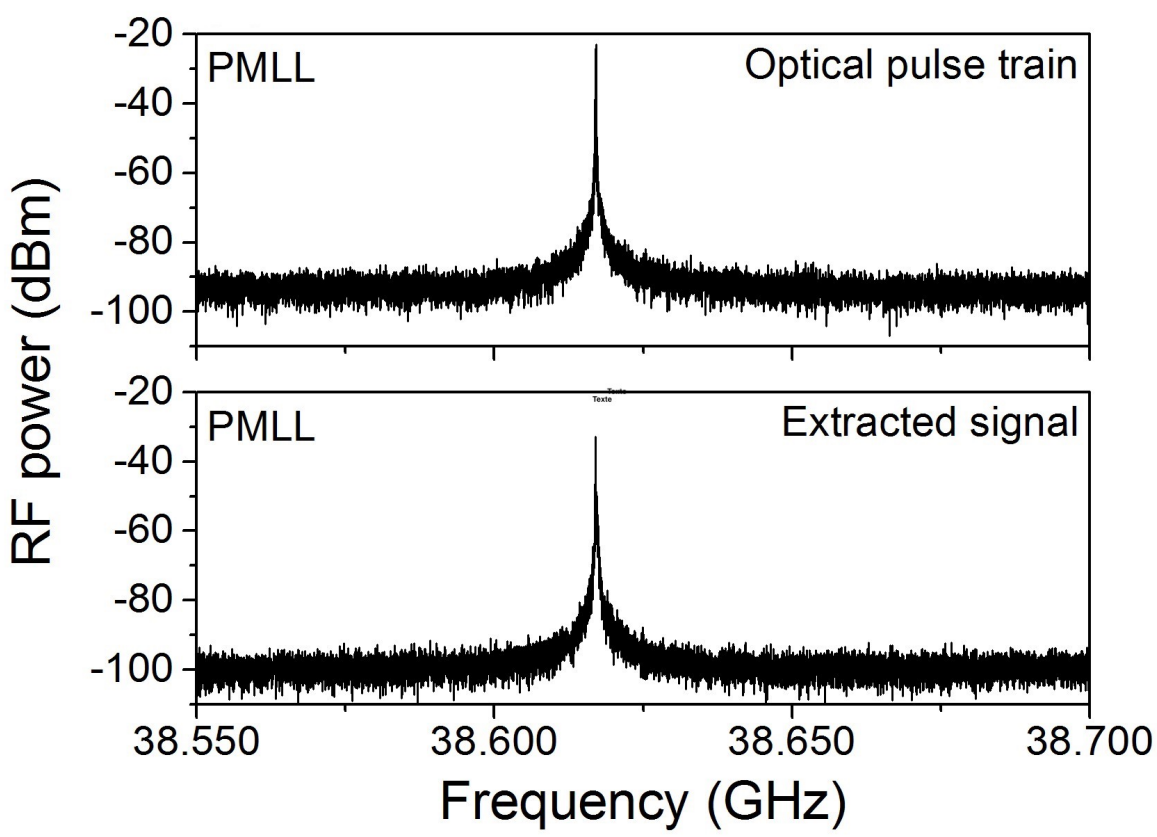

Figure 2. RF spectra of the directly extracted electrical signal from the saturable absorber and the one generated from the optical pulse train respectively

the direct extraction yields a $6.3 \mathrm{~dB}$ lower noise level. On the other hand, the peak power of the extracted signal amounts to $32.8 \mathrm{dBm}$, thus being diminished by $9.8 \mathrm{~dB}$ as compared to the optical pulse train. As shown in Fig. 3(a), the PN power spectral density traces of both signals show a very similar progression, except for the thermal-noise segment (above $10 \mathrm{MHz}$ carrier offset). Nevertheless, the properties of the directly extracted electrical signal from the absorber section essentially conform to the ones obtained for the optical pulse train. The ultra-fast recovery time of QD saturable absorbers enables the electrical signal to perfectly follow the shape of the optical pulse train. The generated photocurrent by the $110 \mu \mathrm{m}$ long saturable absorber is $6.37 \mathrm{~mA}$. According to the definition of the differential DC-to-photocurrent conversion efficiency that is to say the ratio of the average absorber photocurrent to the gain-section DC above threshold, the corresponding value is $16 \%$. An extraction efficiency of $40 \%$ is determined, thus, as well as the differential DC-to-photocurrent conversion efficiency. However, the device used in this work incorporates a seven times shorter absorber section, which is the most critical design parameter of a MLL-based RF-signal source. MLLs, that are designed exclusively for RF-signal generation, differ from those optimized for emission of short optical pulses. A possibility to increase the photocurrent is to vary the bias point of the MLL. Fig. 3(b) depicts the average absorber-photocurrent dependence on the gain current for two different absorber biases $(5 \mathrm{~V}$ and $10 \mathrm{~V})$. An increasing optical power in the cavity, attributed to the increasing gain current, translates to a linearly ascending photocurrent, which is further enhanced at larger reverse biases. However, this progression is not observed for the RF peak power of the extracted signal. Increasing the gain current leads to higher RF peak powers as long the optical pulse energy increases. At a certain point ( $70 \mathrm{~mA}$ in case of $5 \mathrm{~V}$ absorber voltage), the pulse forming ability of the absorber 
(a)

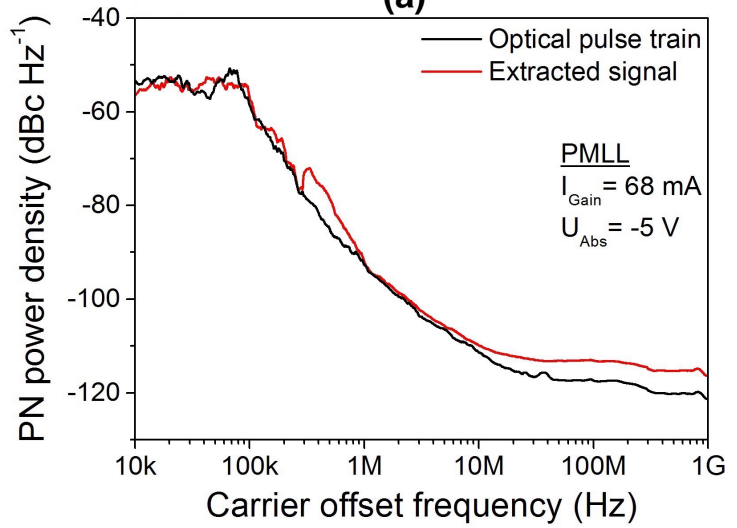

(b)

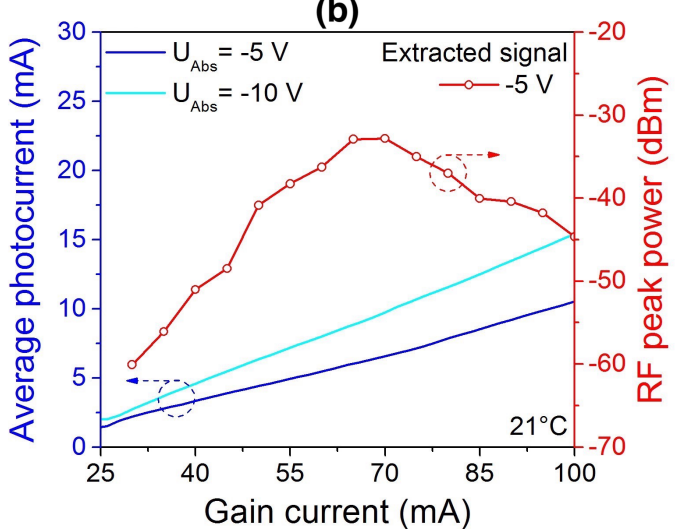

Figure 3. (a) SSB phase-noise traces of the directly extracted electrical signal from the saturable absorber and the one generated from the optical pulse train using a photodiode, respectively. (b) Absorber photocurrent and RF peak-power dependence on gain current and reversed absorber bias. PMLL stands for passive-mode-locking.

saturates. Thus, larger gain currents yield wider pulses accompanied by rising CW background. Consequently, the RF peak power lessens and the carrier linewidth broadens resulting in less stable mode locking, although an increased photocurrent is extracted. By means of variation of the absorber length from $100 \mu \mathrm{m}$ to $250 \mu \mathrm{m}$, an up to $8 \mathrm{~dB}$ enhancement of the RF peak power of extracted electrical signals is achieved. However, the narrow carrier line in electrical spectrum rises then from a wide-frequency pedestal, thus resulting in larger phase-noise power-density values at low carrier offset frequencies and the peak-power benefit vanishes. Nevertheless, also

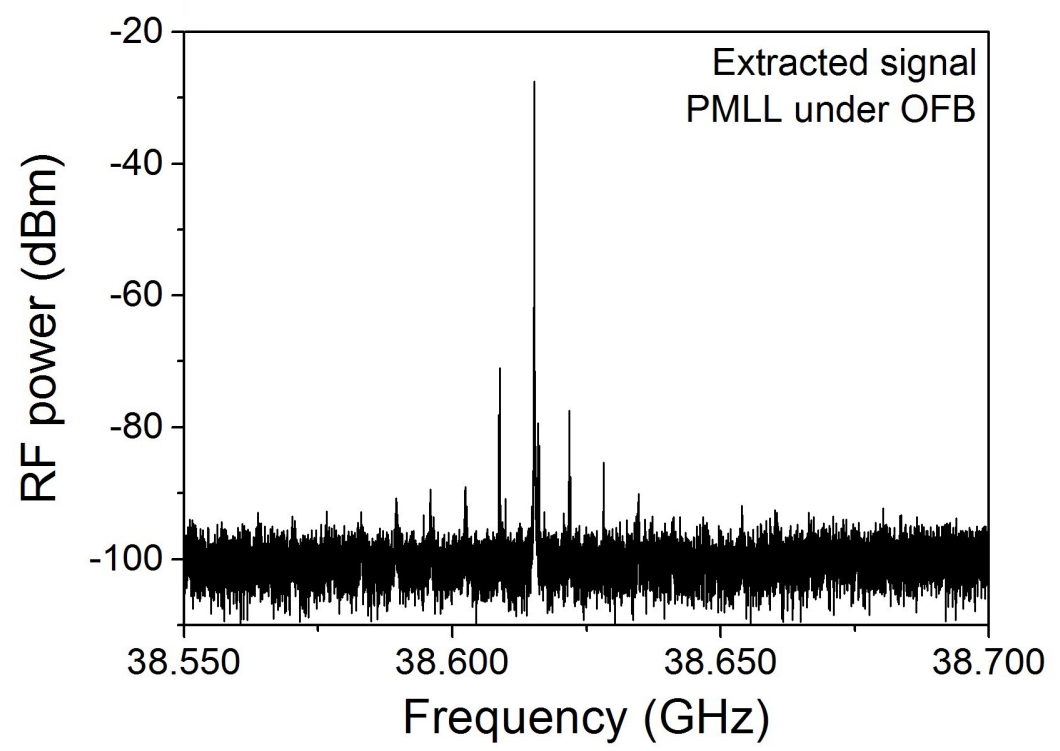

Figure 4. RF spectrum of the extracted electrical signal for the passively MLL under optimal optical feedback loop. PMLL stands for passive-mode-locking.

for MLLs with the best suitable gain-to-absorber length ratio, the still large timing jitter of passive mode-locking translates to the electrical signal. Thus, it was shown in the past that the use of optical feedback (OFB) can strongly reduced the timing jitter ${ }^{20} .{ }^{21}$ Fig. 4 and Fig. 5(a) and Fig. 5(b) shows the RF spectrum and the phase-noise trace for the extracted electrical signal, respectively, while the MLL is operating under the optimal 
(a)
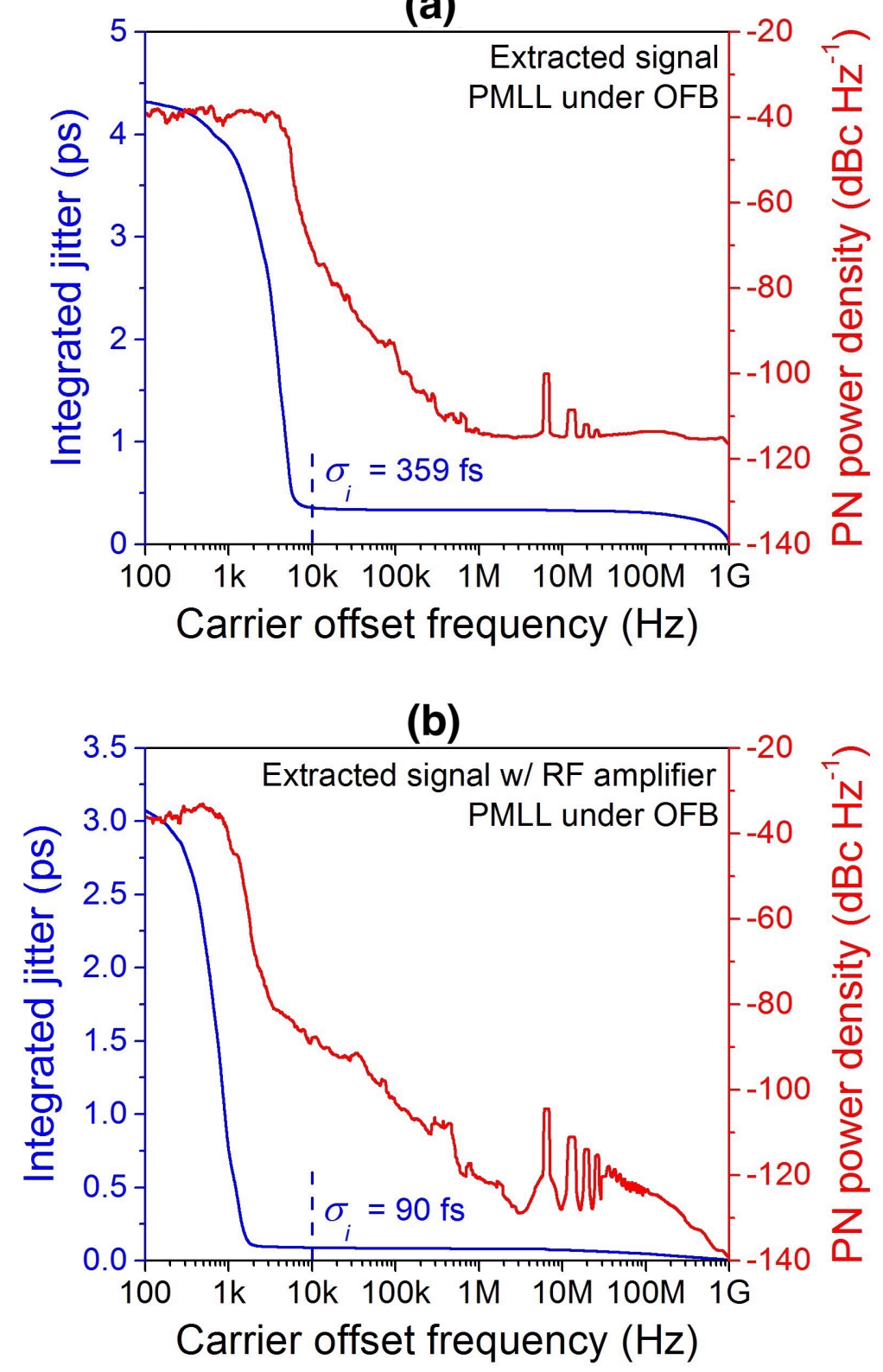

Figure 5. (a) SSB phase-noise trace and integrated timing jitter of the extracted electrical signal for the passively QD MLL under optimal optical feedback loop. (b) The SSB phase-noise trace and integrated timing jitter for the extracted signal that is amplified by $24 \mathrm{~dB}$. PMLL stands for passive-mode-locking.

OFB loop conditions. Let us us stress that the OFB includes a 3-port circulator, a delay line, a polarization controller and a variable attenuator (see Fig. 1). Under optical feedback, a narrow carrier line with an increased peak power of $27.5 \mathrm{dBm}$ and OFB typical sidebands at $6.5 \mathrm{MHz}$ distance are observed. The SNR and sidebandsuppression-ratio (SBSR) are $68.2 \mathrm{~dB}$ and $43.5 \mathrm{~dB}$, respectively. Compared to the optical pulse train, a slightly wider white-noise region with a corner frequency of $5 \mathrm{kHz}(3 \mathrm{kHz}$ for the optical signal) is present. The 3$\mathrm{dB}$ linewidth and the integrated timing jitter are $3.25 \mathrm{kHz}$ and $359 \mathrm{fs}(1.92 \mathrm{kHz}$ and $219 \mathrm{fs}$ for the optical signal), respectively. The potential of the QD MLL under OFB operating as a RF-signal source is pointed out in Fig. 5(b). The extracted electrical signal is amplified by means of a 24 -dB broadband power amplifier. A 
corresponding integrated timing jitter as low as $90 \mathrm{fs}$ is unveiled, that is to say a reduction by a factor of four. Hence, the minimal phase-noise power spectral density of the extracted signal is not given by the MLL itself, but presently limited by extraction capabilities.

\section{NONLINEAR DYNAMICS OF QD LASERS}

This section reports on comparative optical feedback experiments performed on two InAs/GaAs QD Fabry-Perot (FP) lasers. The QD lasers share the same active regions and do not exhibit a two-state lasing dynamics where ES and GS lasing can take place simultaneously. ${ }^{22}$ Instead they doc emit either exclusively on the GS or the ES. Overall, the experiments show richer feedback dynamics in the ES QD laser as compared to GS lasers in which a chaos-free operation is reported.

\section{QD laser structure}

The active region of both devices is similar to the one described in the previous section. It is still based on a dot-in-well structure, including $10 \mathrm{InAs}$ dot sheets grown by MBE. FP lasers are left as-cleaved and cavity lengths are both $1 \mathrm{~mm}$ long while the ridge waveguide etched through the active region is $2 \mu \mathrm{m}$ wide. In this work, a free-space optical feedback platform is used. Details of the experimental setup can be found elsewhere. ${ }^{23}$ In the experiments, the feedback strength $r_{\text {ext }}$ is controlled by a free-space variable attenuator. The lens coupling ratio is similar for both lasers, allowing a maximal feedback ratio up to $60 \%$. By moving the position mirror, the external cavity length was varied from 2 to $50 \mathrm{~cm}$. On the right side of the laser, the emission is coupled by an AR coated lens-end fiber and passes through an optical isolator for analysis. On the detection path, an OSA, an ESA and a sampling oscilloscope are connected simultaneously to visualize the signals characteristics. The sampling scope used in this experiment has a $6 \mathrm{GHz}$ bandwidth and a $20 \mathrm{~Gb} / \mathrm{s}$ sampling rate. The relaxation oscillations frequencies $f_{R O}$ for both lasers are of the order of few $\mathrm{GHz}$.

\section{GS QD laser dynamics}

Fig. 6 shows the optical and RF mappings measured for the GS QD laser for a short external cavity length of 2 $\mathrm{cm}$ long $\left(f_{R O} \times \tau \approx 0.2\right)$ and for two different bias levels. Fig. 6(a) and Fig. 6(c) show the evolution of FP modes around the gain peak as a function of $r_{\text {ext }}$. The maps reveal that the optical spectra are not stable regardless the value of the bias current, exhibiting some broadenings of the modes. Over the whole emission spectrum, two sets of modes are interacting inversely: while one set is strongly lasing, the other is rejected with a suppression ratio above $15 \mathrm{~dB}$ for the modes around the gain peak. This effect observed for the short-cavity regime may be attributed to an antiphase modal dynamics but further investigations are required at this stage to confirm this assumption. While no evidence of such dynamics was observed in the RF spectral maps depicted in Fig. 6(b) and Fig. 6(d), it is however possible to identify in the electrical domain a trace of a weak peak $(\approx-80 \mathrm{dBm})$ observed around $4 \mathrm{GHz}$. Given that an external cavity length of $2 \mathrm{~cm}\left(f_{\text {ext }}=7.5 \mathrm{GHz}\right)$, this peak corresponds to the double round-trip within the external cavity which may be induced by the reflection of the delayed field from the cleaved facet.

Figures 7 shows that the RF spectral mappings and the corresponding RF spectra at $1.5 \times$ and $1.7 \times I_{t h}$ under free-running $\left(r_{e x t}=0\right)$ and for the maximal optical feedback strength $\left(r_{e x t}=76 \%\right)$ of GS QD laser for a longer external cavity length of $50 \mathrm{~cm}\left(f_{R O} \times \tau \approx 10\right)$. In this case, more external cavity modes are involved in the laser dynamics. Thus, results show that the GS laser remains first perfectly stable then follows a route driven by quasi-periodic oscillations but without reaching any chaotic state. The absence of chaotic oscillations in this GS QD laser even at very large feedback strength is fundamentally explained by the very large damping factor $^{24} \cdot{ }^{23}$

\section{ES dynamics}

The dynamics of ES QD laser is found much richer. Fig. 8(a) and Fig. 8(c) show the RF mappings at $1.5 \times I_{t h}$ under free-running $\left(r_{\text {ext }}=0\right)$ and for the maximal optical feedback strength $\left(r_{\text {ext }}=55 \%\right)$ considering 2 and 50 $\mathrm{cm}$ long external cavity lengths respectively. Fig. 8(b) and Fig. 8(d) depict the corresponding RF spectra at maximal feedback $\left(r_{e x t}=55 \%\right)$ that are compared to the free-running case $\left(r_{e x t}=0\right)$. In Fig. 8(a) and Fig. 8(b), the strong beating at $3.8 \mathrm{GHz}$ still corresponds to the signature of the double roundtrip within the external 

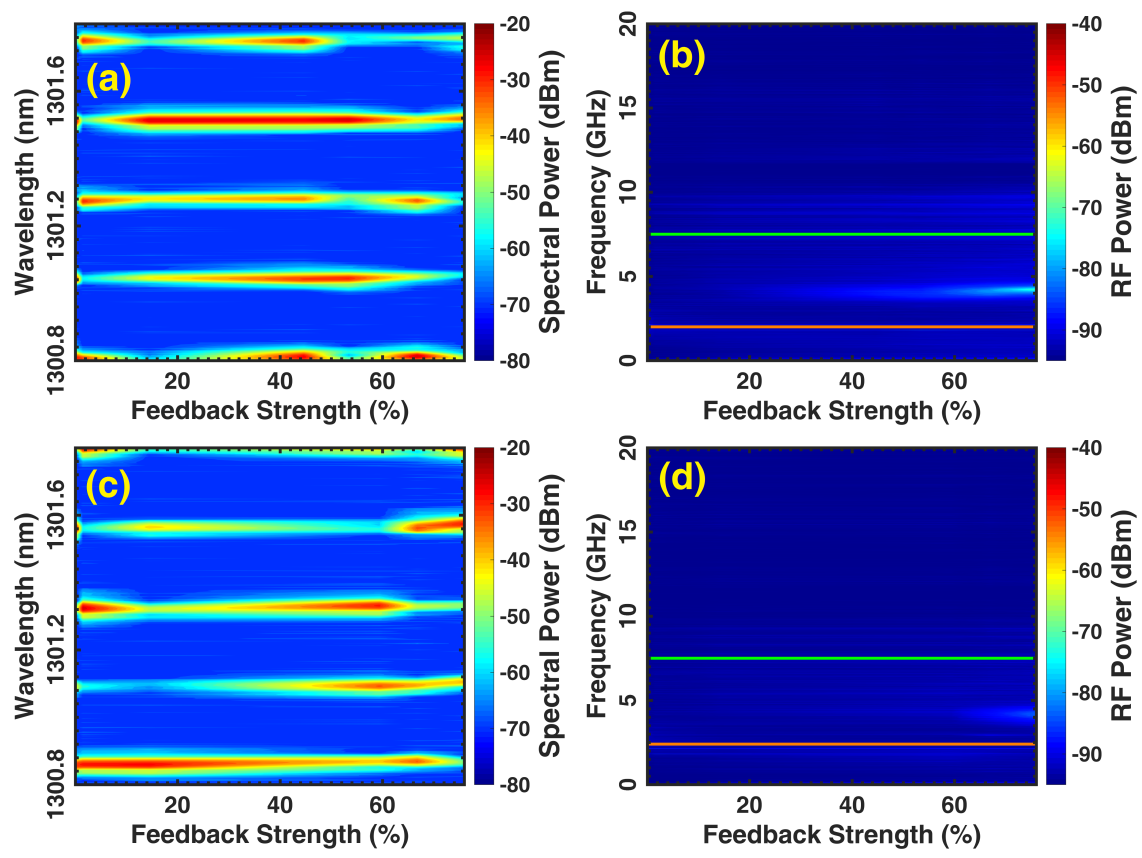

Figure 6. Optical and RF mappings of the GS QD laser. The external cavity length is $2 \mathrm{~cm}$ long. The bias currents are at $1.5 \times((\mathrm{a})$ and $(\mathrm{b}))$ and $1.7 \times I_{t h}((\mathrm{c})$ and $(\mathrm{d}))$. The green and the orange horizontal solid lines mark the frequencies $f_{\text {ext }}$ and $f_{R O}$ respectively.
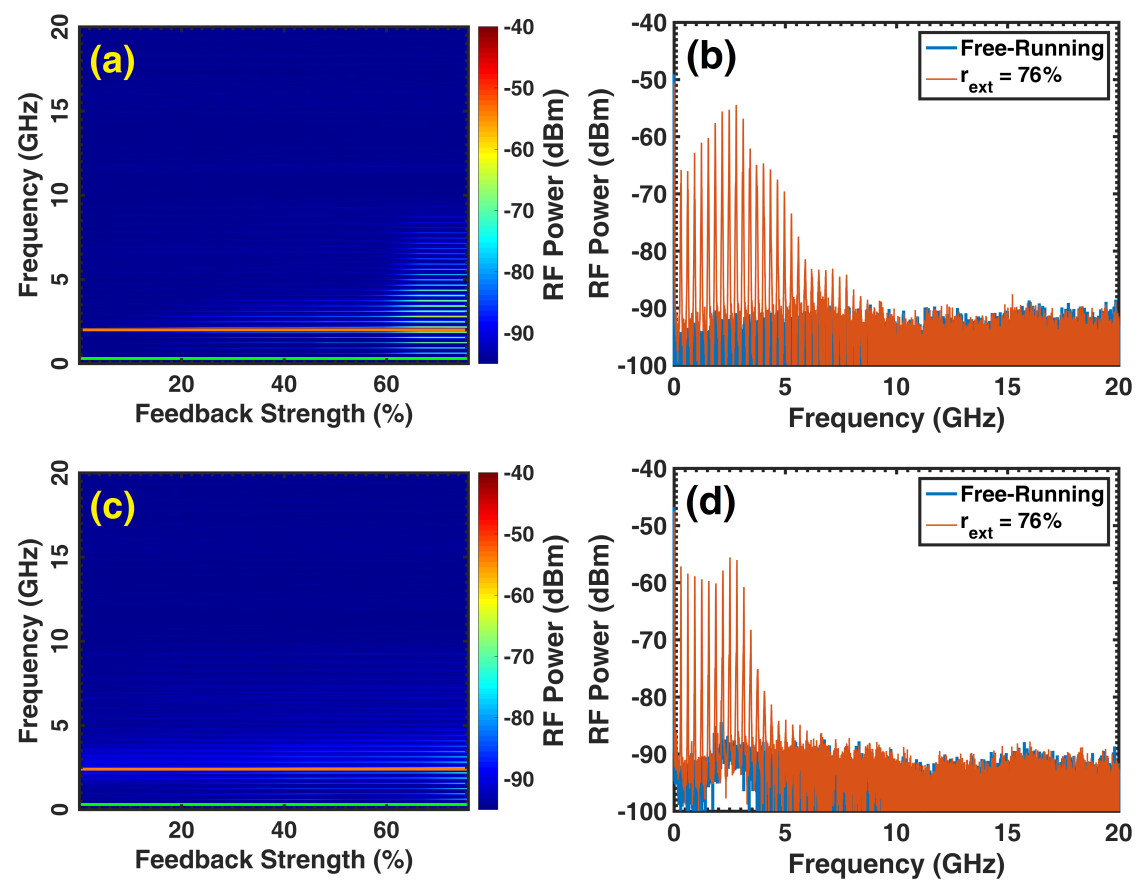

Figure 7. RF mappings ((a), (c)) and RF spectra ((b), (d)) under free-running $\left(r_{\text {ext }}=0\right)$ and for the maximal optical feedback strength $\left(r_{\text {ext }}=76 \%\right)((\mathrm{b}),(\mathrm{d}))$ of GS QD laser. The external cavity length is $50 \mathrm{~cm}$ long. Bias currents are at $1.5 \times((\mathrm{a}),(\mathrm{b}))$ and $1.7 \times I_{t h}((\mathrm{c}),(\mathrm{d}))$. The green and the orange horizontal solid lines mark the frequencies $f_{\text {ext }}$ and $f_{R O}$ respectively.

cavity, similar to what observed with the GS QD laser. However, in such case, the oscillation peak is enhanced well beyond the noise level, and a high pedestal begins to develop at around $40 \%$ as opposed to the GS laser for 
which the oscillations were much weaker due to the stronger damping rate. By increasing the external cavity up to $50 \mathrm{~cm}$, a typical destabilization scenario is retrieved hence the ES laser starts oscillating under low feedback and then falls quickly into chaos through quasi-periodic oscillations.

Although the two lasers are made from the same active medium, their responses to the optical feedback are found not much alike. The GS laser does not display chaotic pulsations whatever the external cavity length and the optical feedback strength. In contrast, the ES laser exhibits richer nonlinear dynamics with both periodic and chaotic oscillations. Such a difference is attributed to the very large damping factor of the GS laser preventing any chaotic oscillations even at the largest feedback ratios. The ES laser follows a more traditional route towards the chaos while the GS one appears less sensitive to chaos and exhibits longitudinal modes dynamics instead. As a conclusion, these results provide useful guidelines for featuring new QD lasers. While the more stable GS laser can be important for the development of isolator-free and all-optical logic function transmitters benefiting from its great resistance against feedback, the ES laser, on the other hand is suitable for applications such as lidars, radars, and high-speed random bit generations.
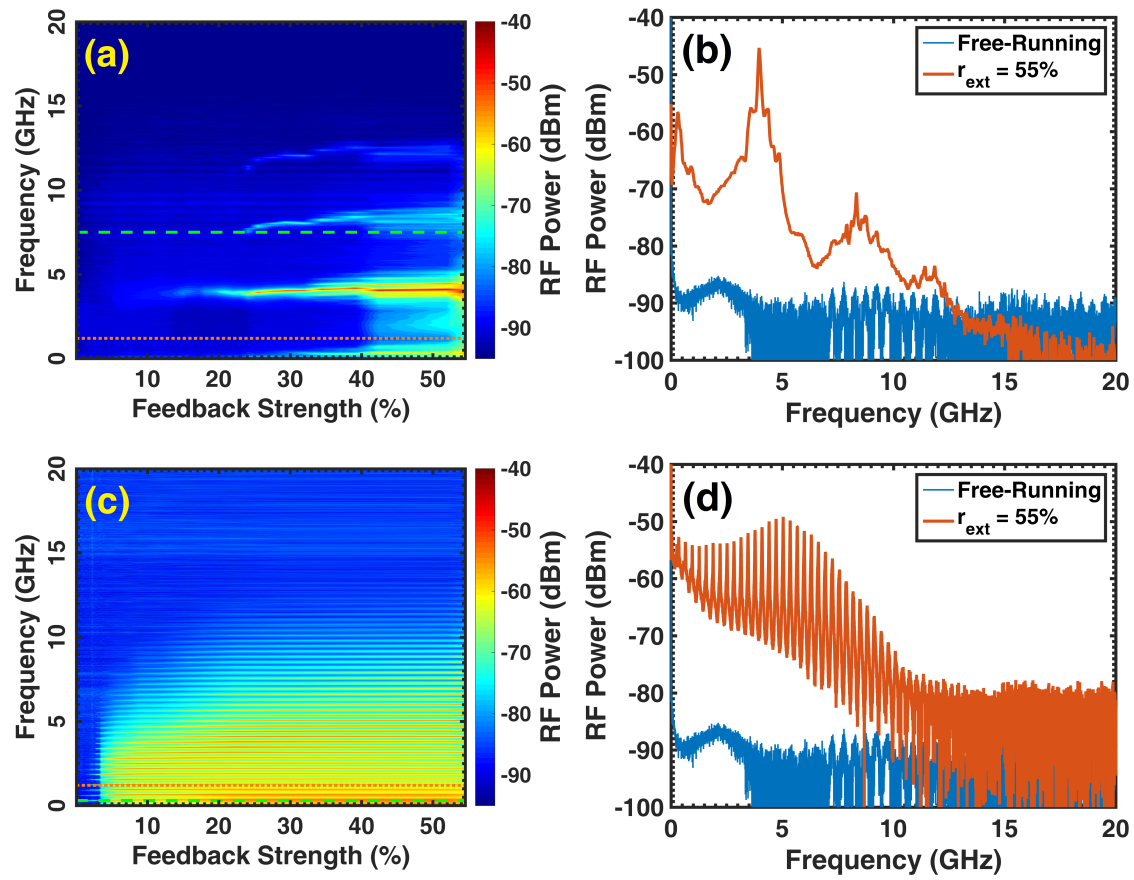

Figure 8. RF mappings of the ES QD laser biased at $1.5 \times I_{t h}$ under free-running and for the maximal optical feedback strength $\left(r_{\text {ext }}=76 \%\right)$ considering 2 and $50 \mathrm{~cm}$ long external cavity ((a) and (c)); RF spectra under free-running $\left(r_{\text {ext }}=0\right)$ and for the maximal optical feedback strength $\left(r_{\text {ext }}=55 \%\right)((\mathrm{b})$ and $(\mathrm{d}))$.

\section{CONCLUSIONS}

This paper reports on the recent achievements in the ultrafast and nonlinear dynamics properties of InAs/GaAs QD lasers. Passive mode-locking for microwave, millimeter-wave and $\mathrm{THz}$ signal generation is first discussed then multimode optical feedback dynamics of QD lasers emitting on different lasing states. The former show that passively QD MLLs exhibit a great potential to reach high repetition frequency tuning. With an optimal $\mathrm{OFB}$, timing jitter as low as $90 \mathrm{fs}$ is also reported. The later unveils that QD lasers made from the same active material but emitting on different lasing states can produce different responses to external perturbations. In particular, a chaos free operation is observed in the GS QD laser which a very important feature for designing isolator-free optical light sources. ${ }^{23}$ Overall, the results presented in this work sheds the light on future emerging technologies for radar systems, wireless communications and high-speed optical data communications. 


\section{ACKNOWLEDGMENTS}

This work is supported by the SFB 787 of DFG in the framework of the SFB787, the Institut Mines Tlcom (IMT) through the Futurs \& Ruptures program, and by the European Office of Aerospace Research and Development under Grant FA9550-15- 1-0104.

\section{REFERENCES}

[1] Bimberg, D., "Quantum dot based nanophotonics and nanoelectronics," Electron. Lett. 44, 390 (2008).

[2] Crowley, M. T., Naderi, N. A., Su, H., Grillot, F., and Lester, L. F., [GaAs-Based Quantum Dot Lasers, in Advances in Semiconductor Lasers], Academic Press (2012).

[3] Eisenstein, G. and Bimberg, D., [Green Photonics and Electronics], Springer (2017).

[4] Shchekin, O. and Deppe, D., "1.3 $\mu \mathrm{m}$ InAs quantum dot laser with T0=161 K from 0 to 80 degrees c," Appl. Phys. Lett. 80, 3277 (2002).

[5] Arsenijević, D. and Bimberg, D., "Quantum-dot lasers for 35 gbit/s pulse-amplitude modulation and 160 gbit/s differential quadrature phase-shift keying," Proc. SPIE 9892 (2016).

[6] "Ranovus announces availability of world's first quantum dot multi-wavelength laser and silicon photonics platform technologies to create a new cost and power consumption paradigm for dci market," http://ranovus.com/worlds-first-quantum-dot-multi-wavelength-laser-and-silicon-photonics-platformtechnologies-for-dci-market/. (2016).

[7] Malinsa, D. B., Gomez-Iglesias, A., White, S. J., Sibbett, W., and Miller, A., "Ultrafast electroabsorption dynamics in an InAs quantum dot saturable absorber at $1.3 \mu \mathrm{m}, "$ Appl. Phys. Lett. 89, 171111 (2006).

[8] Gomis-Bresco, J., Dommers-Vlkel, S., Schps, O., Kaptan, Y., Dyatlova, O., Bimberg, D., and Woggon, U., "Time-resolved amplified spontaneous emission in quantum dots," Appl. Phys. Lett. 97, 251106 (2010).

[9] Wang, C., Lingnau, B., Lüdge, K., Even, J., and Grillot, F., "Enhanced dynamic performance of quantum dot semiconductor lasers operating on the excited state," IEEE J. Quantum Electron. 50, 723 (2014).

[10] Arsenijević, D., Schliwa, A., Schmeckebier, H., Stubenrauch, M., Spiegelberg, M., Bimberg, D., Mikhelashvili, V., and Eisenstein, G., "Comparison of dynamic properties of ground- and excited-state emission in p-doped InAs/GaAs quantum-dot lasers," Appl. Phys. Lett. 104, 181101 (2014).

[11] VanDijk, F. et al., "Quantum dash mode-locked lasers for millimeter wave signal generation and transmission," in Annual Meeting of the IEEE Photonics Society (2010).

[12] Stohr, A. et al., "Millimeter-wave photonic components for broadband wireless systems," IEEE Transactions on Microwave Theory and Techniques 58, 3071 (2010).

[13] Knox, W., "Ultrafast technology in telecommunications," IEEE J. Quantum Electron. 6, 1273 (2000).

[14] Hillerkuss, D. et al., "26 tbit/s line-rate super-channel transmission utilizing all-optical fast fourier transform processing," Nature Photonics 5, 364 (2011).

[15] Dorrer, C., "High-speed measurements for optical telecommunication systems," IEEE J. Quantum Electron. 12, 843 (2006).

[16] Qi, X. and Liu, J., "Photonic microwave applications of the dynamics of semiconductor lasers," IEEE J. of Selected Topics in Quantum Electron. 17, 1198 (2011).

[17] Novak, D., Zaheer Ahmed, R. B. W., and Tucker, R. S., "Signal generation using pulsed semiconductorlasers for application in millimeter-wave wireless links," IEEE Transactions on Microwave Theory and Techniques 43, 2257 (1995).

[18] Deppe, D., Huang, H., and Shchekin, O., "Modulation characteristics of quantum-dot lasers: the influence of p-type doping and the electronic density of states on obtaining high speed," IEEE J. Quantum Electron. 38, 1587 (2002).

[19] Mee, J. K., Raghunathan, R., Wright, J. B., and Lester, L. F., "Device geometry considerations for ridge waveguide quantum dot mode-locked lasers," Journal of Physics D: Applied Physics 47, 233001 (2014).

[20] Lin, C.-Y., Grillot, F., Naderi, N. A., Li, Y., Raghunathan, R., and Lester, L. F., "Microwave characterization and stabilization of timing jitter in a quantum-dot passively mode-locked laser via external optical feedback," IEEE J. of Selected Topics in Quantum Electron. 17, 1311 (2011). 
[21] Arsenijevic, D., Kleinert, M., and Bimberg, D., "Phase noise and jitter reduction by optical feedback on passively mode-locked quantum-dot lasers," Appl. Phys. Lett. 103, 231101 (2013).

[22] Veselinov, K., Grillot, F., Cornet, C., Even, J., Bekiarski, A., Gioannini, M., and Loualiche, S., "Analysis of the double laser emission occurring in $1.55 \mu \mathrm{m}$ InAs/InP (113)b quantum-dot lasers," IEEE J. Quantum Electron. 43, 810 (2007).

[23] Huang, H., Lin, L.-C., Chen, C.-Y., Asenijevic, D., Bimberg, D., Lin, F.-Y., and Grillot, F., "Multimode optical feedback dynamics in InAs/GaAs quantum dot lasers emitting exclusively on ground or excited states: transition from short- to long-delay regimes," Optics Express 26, 1743 (2018).

[24] O'Brien, D., Hegarty, S., Huyet, G., J. McInerney, T. K., Laemmlin, M., Bimberg, D., Ustinov, V., A. Zhukov, S. M., and Kovsh, A., "Feedback sensitivity of $1.3 \mu \mathrm{m}$ InAs/GaAs quantum dot lasers," Electron. Lett. 39, 1819 (2003). 